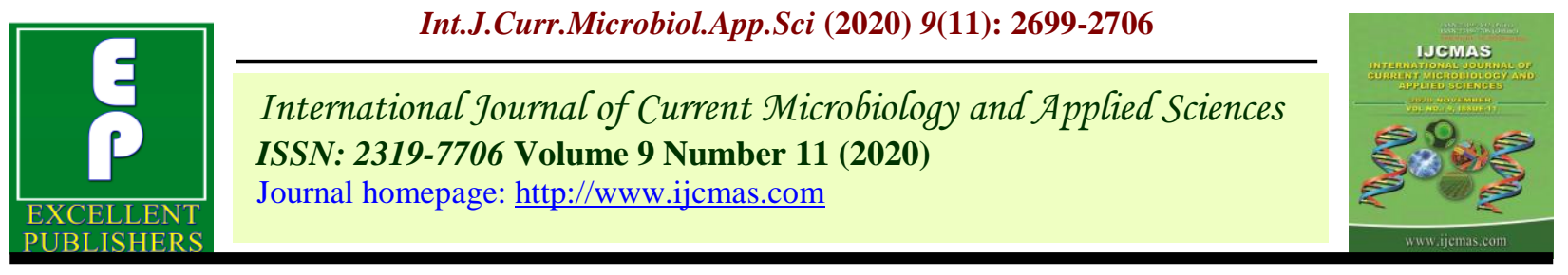

Original Research Article

https://doi.org/10.20546/ijcmas.2020.911.327

\title{
Assessment of Post-emergence Herbicides in Direct Seeded Rice (Oryza sativa L.) under Chhattisgarh Plain
}

\author{
R. K. Satyaraj Guru* and Sanjay K. Dwivedi \\ Department of Agronomy, Indira Gandhi Krishi Vishwavidyalaya, \\ Raipur 492012 (C.G.), India \\ *Corresponding author
}

\begin{tabular}{l} 
K e y w o r d s \\
$\begin{array}{l}\text { Direct seeded rice, } \\
\text { Post-emergence } \\
\text { herbicides, Number } \\
\text { of filled grains per } \\
\text { panicle and Weed } \\
\text { control index }\end{array}$ \\
Article Info \\
$\begin{array}{l}\text { Accepted: } \\
\text { 20 October } 2020 \\
\text { Available Online: } \\
\text { 10 November } 2020\end{array}$ \\
\hline
\end{tabular}

Keywords

Direct seeded rice Post-emergence herbicides, Number control index

Article Info

Accepted:

10 November 2020

\section{A B S T R A C T}

An experiment was conducted to study the efficacy of post-emergence herbicides in direct seeded rice at I.G.K.V, Raipur, Chhattisgarh during Kharif2017 and 2018, having eleven treatments replicated thrice in randomized block design. The data revealed that the total dry matter of grasses were 79.4 and $78.4 \%$ higher during 2018 as compare to 2017 at 30 and 60 days after sowing, respectively. However, the total dry matter of broad leaved weeds were 44.9 and $77.4 \%$ higher during 2017 as compare to 2018at 30 and 60 days after sowing, respectively. The weed free $(20 \& 40$ DAS) $\left(T_{10}\right)$ treatment and weedy check $\left(T_{11}\right)$ treatment recorded the highest and lowest value with respect to crop dry matter accumulation, weed control index, number of filled grains per panicle, grain and straw yield, respectively. Among the herbicide treatments, the highest yield attributes, grain and straw yield was found under the application of bispyribac sodium $2 \%+2,4$ - D sodium salt $54.3 \% \mathrm{SP}$ with adjuvant $\left(30.0+814.5 \mathrm{~g} \mathrm{ha}^{-1}\right)\left(\mathrm{T}_{6}\right)$, which was at par with the weed free $(20 \& 40$ DAS $)\left(T_{10}\right)$ treatment.

\section{Introduction}

Rice is the most important and extensively grown crop in Chhattisgarh, known as the "Rice bowl of central India" (Mooventhan et $a l ., 2015)$. The state is comprised of three agro-ecological zones i.e. Chhattisgarh plain, bastar plateau and northern hill region and show immense variations in terms of soil topography, rainfall intensity and distribution, irrigation and adoption of agricultural production system and thus show greater variation in the productivity of rice in these regions. Rice is intake as the major source of carbohydrate in meal, apart from this a number of processed food items prepared from rice grain flatters the taste buds of inhabitants. The state accounts for $3.82 \mathrm{M}$ ha area with the production of $4.80 \mathrm{MT}$ and average productivity of $1.32 \mathrm{t} \mathrm{ha}^{-1}$ (Anon., 
2015). In addition to that increasing transplanting cost and declining profitability of transplanted rice production system coupled with less labour requirement and low cost of cultivation under direct seeding encouraged rice farmers to shift from transplanting to direct seeding (Subbaiah et al., 1999; Mishra and Singh, 2011). Direct seeding of rice (DSR) is possible, provided there is adequate weed control methods are available to keep the crop free from weeds (Rao and Nagamani, 2007), however in absence of proper weed control, rice yields are reduced by 35-100 per cent in DSR (Kumar et al., 2008). Manual removal of weeds is labour intensive, tedious, backbreaking and does not ensure weed removal at the critical stage of crop-weed competition due to non-availability of labours and sometimes bad weather condition, which does not allow labours to move in the field. Thus, herbicides are considered to be an alternative to hand weeding (Singh et al., 2006). Jacob et al., (2014) reported that the major advantage with herbicidal control of weeds in DSR is the reduction in the cost of cultivation. Most of the herbicides that are recommended for direct seeded rice are applied as pre-emergence to control weeds during the initial period. The weeds that emerge during the later periods of crop growth cycle poses utmost threat to crop growth. Therefore, the present investigation evaluated the efficacy of suitable postemergence herbicides for effective control of dominant and diversified weed flora in direct seeded rice crop.

\section{Materials and Methods}

The field experiment was conducted during Kharif 2017 and 2018 at I.G.K.V, Raipur $\left(21^{\circ} 23^{\prime} \mathrm{N}, 81^{\circ} 71^{\prime} \mathrm{E}\right.$ and $290 \mathrm{~m}$ above mean sea level), Chhattisgarh. The soil was neutral in reaction $(\mathrm{pH}$ 6.8) and low in available nitrogen, medium in phosphorus and high in potassium. Eleven treatments viz. bispyribac sodium $2 \%(\mathrm{BS})+2,4-\mathrm{D}$ sodium salt $54.3 \%$ SP (DSS) $\left(20.0+543.0 \mathrm{~g} \mathrm{ha}^{-1}\right)\left(\mathrm{T}_{1}\right), \mathrm{BS}+2,4-$ DSS $\left(25.0+678.75 \mathrm{~g} \mathrm{ha}^{-1}\right)\left(\mathrm{T}_{2}\right), \mathrm{BS}+2,4-$ DSS $\left(30.0+814.5 \mathrm{~g} \mathrm{ha}^{-1}\right)\left(\mathrm{T}_{3}\right), \mathrm{BS}+2$, 4DSS with adjuvant (WA) $\left(20.0+543.0 \mathrm{~g} \mathrm{ha}^{-}\right.$ $\left.{ }^{1}\right)\left(\mathrm{T}_{4}\right), \mathrm{BS}+2,4-\mathrm{DSS}(\mathrm{WA})(25.0+678.75 \mathrm{~g}$ $\left.\mathrm{ha}^{-1}\right)\left(\mathrm{T}_{5}\right), \mathrm{BS}+2,4-\mathrm{DSS}$ (WA) $(30.0+814.5$ $\left.\mathrm{g} \mathrm{ha}^{-1}\right)\left(\mathrm{T}_{6}\right)$, bispyribac sodium 10\% SC @ 20 g a.i. ha ${ }^{-1}\left(\mathrm{~T}_{7}\right), 2$, 4- D Ethyl ester $38 \%$ EC $(34 \% \mathrm{~W} / \mathrm{W}) \quad(\mathrm{EE}) \quad\left(850 \mathrm{~g} \quad \mathrm{ha}^{-1}\right) \quad\left(\mathrm{T}_{8}\right)$, penoxsulam $21.7 \% \mathrm{SC}\left(22.5 \mathrm{~g} \mathrm{ha}^{-1}\right)\left(\mathrm{T}_{9}\right)$, weed free (20 and 40 DAS) $\left(\mathrm{T}_{10}\right)$ and weedy check $\left(T_{11}\right)$ were taken during both years. These eleven treatments were laid out in randomized block design with three replications. Pre-germinated seeds of medium duration rice variety 'Indira Rajeshwari (IGKV R 1)' were line sown on non-puddled levelled field on $20^{\text {th }}$ and $30^{\text {th }}$ June of 2017 and 2018, respectively, with a seed rate of 80 $\mathrm{kg} \mathrm{ha}{ }^{-1}$. The crop was fertilized with 100:60:40 N: $\mathrm{P}_{2} \mathrm{O}_{5}: \mathrm{K}_{2} \mathrm{O} \mathrm{kg} \mathrm{ha}^{-1}$, respectively during both years and $50 \%$ nitrogen, entire dose of phosphorous and potassium was applied as basal. The remaining $50 \%$ of the nitrogen was top dressed at two equal splits at tillering and panicle initiation stage. The herbicides were sprayed at 2-3 leaf stage of weeds by using knapsack sprayer mixed with water 500 litre $\mathrm{ha}^{-1}$. All herbicides were applied at 18 DAS. All other agronomic and plant protection measures were adopted as per the recommended packages of IGKV, Raipur, Chhattisgarh, India. The data on weed density and weed dry weight (at 30 and 60 DAS) were recorded with the help of quadrat $(0.5 \mathrm{x}$ $0.5 \mathrm{~m})$. Data on yield attributes and grain yield were recorded. The weed control index (at 30 and 60 DAS) was worked out taking into consideration the reduction in weed population in treated plot over weed population in weedy check plot as suggested by Kumar et al., (2019)

Weed Control Index $(W C I)=\frac{W P_{C}-W P_{T}}{W P_{C}} \times 100$ 
Where, $\mathrm{WP}_{\mathrm{C}}=$ Weed population in control plot

$\mathrm{WP}_{\mathrm{T}}=$ Weed population in treated plot

\section{Results and Discussion}

\section{Weed density and dry weight}

The most dominant weed species found in the experimental plot were Echinochloa colona and Ischaemum rugosum among grassy weeds; Cyperusiria among sedges; Alternanthera sessilis and Eclipta alba among broad-leave weeds. The weed density and dry weight of grassy weeds, sedges and broad-leaf weeds at 30 and 60 DAS during both years were presented in the Table 1 and 2, respectively. At $30 \mathrm{DAS}$, the highest total weed density and dry weight were recorded under the weedy check $\left(\mathrm{T}_{11}\right)$ treatment. Among the herbicide treatments, the lowest weed density and dry weight was found under the application of $\mathrm{BS}+2$, 4- DSS (WA) (30.0 $\left.+814.5 \mathrm{~g} \mathrm{ha}^{-1}\right)\left(\mathrm{T}_{6}\right)$. It also recorded the lower weed density $(88.9 \%$ in 2017 and $87.4 \%$ in 2018) and dry weight (98.5\% and $96.5 \%)$ as compare to the weedy check $\left(\mathrm{T}_{11}\right)$ treatment. At 60 DAS, each treatments noticed increase in weed density and dry weight as compare to 30 DAS. Among the herbicide treatments, the lowest weed density and dry weight was found under the application of BS+ 2, 4- DSS (WA) $\left(30.0+814.5 \mathrm{~g} \mathrm{ha}^{-1}\right)\left(\mathrm{T}_{6}\right)$ followed by $\mathrm{BS}+2$, 4- DSS (WA) $\left(25.0+678.75 \mathrm{~g} \mathrm{ha}^{-1}\right)$ $\left(\mathrm{T}_{5}\right)$. During both years, at 30 and $60 \mathrm{DAS}$, the density and dry weight of grassy weeds were higher during 2018 compare to 2017 and vice versa in case of broad-leaved weeds.

\section{Crop dry matter accumulation and Weed control index}

The highest crop dry matter accumulation was noticed under the weed free (20 \& 40 DAS) $\left(\mathrm{T}_{10}\right)$ treatment and lowest was found under the weedy check $\left(T_{11}\right)$ treatment, at 60 DAS during both years (Table 3). Among the herbicide treatments, the highest dry matter accumulation of crop was recorded under the application of BS+ 2, 4- DSS (WA) $(30.0+$ $\left.814.5 \mathrm{~g} \mathrm{ha}^{-1}\right)\left(\mathrm{T}_{6}\right)$ followed by BS+2, 4- DSS (WA) $\left(25.0+678.75 \mathrm{~g} \mathrm{ha}^{-1}\right)\left(\mathrm{T}_{5}\right), \mathrm{BS}+2,4-$ DSS $\left(30.0+814.5 \mathrm{~g} \mathrm{ha}^{-1}\right)\left(\mathrm{T}_{3}\right)$ and $\mathrm{BS}+2,4-$ DSS (WA) $\left(20.0+543.0 \mathrm{~g} \mathrm{ha}^{-1}\right)\left(\mathrm{T}_{4}\right)$, which were at par with the weed free (20 \& 40 DAS) $\left(\mathrm{T}_{10}\right)$ treatment.

Among the herbicide treatments, the highest and lowest weed control index (WCI) was recorded under the application of $\mathrm{BS}+2$, 4DSS (WA) $\left(30.0+814.5 \mathrm{~g} \mathrm{ha}^{-1}\right)\left(\mathrm{T}_{6}\right)$ and $2,4-$ DEE $\left(850 \mathrm{~g} \mathrm{ha}^{-1}\right)\left(\mathrm{T}_{8}\right)$, respectively at 30 and 60 DAS, during both years (Fig. 1a and 1b). Higher weed control index was recorded during 2017 as compare to 2018, due to lower weeds infestation.

\section{Yield and yield attributes}

The weed free $(20 \& 40$ DAS $)\left(\mathrm{T}_{10}\right)$ treatment recorded highest number of filled grains per panicle (132.8 and 126.7), grain yield (59.2 and $57.5 \mathrm{q} \mathrm{ha}^{-1}$ ) and straw yield (72.3 and $70.8 \mathrm{q} \mathrm{ha}^{-1}$ ) and lowest number of unfilled grains per panicle (9.50 and 11.0) during both years, due to less infestation of weed flora (Table 3).

It was found at par with the application of $\mathrm{BS}+2$, 4- DSS (WA) $\left(30.0+814.5 \mathrm{~g} \mathrm{ha}^{-1}\right)$ $\left(\mathrm{T}_{6}\right), \mathrm{BS}+2,4-\mathrm{DSS}$ (WA) $(25.0+678.75 \mathrm{~g}$ $\left.\mathrm{ha}^{-1}\right)\left(\mathrm{T}_{5}\right), \mathrm{BS}+2,4-$ DSS $\left(30.0+814.5 \mathrm{~g} \mathrm{ha}^{-}\right.$ $\left.{ }^{1}\right)\left(\mathrm{T}_{3}\right), \mathrm{BS}+2,4-\mathrm{DSS}(\mathrm{WA})(20.0+543.0 \mathrm{~g}$ $\left.\mathrm{ha}^{-1}\right)\left(\mathrm{T}_{4}\right)$ during both years.

The lowest number of filled grains per panicle (79.3 and 73.0), grain yield (29.0 and $28.4 \mathrm{q}$ $\mathrm{ha}^{-1}$ ), straw yield (38.4 and $38.2 \mathrm{q} \mathrm{ha}^{-1}$ ) and highest number of unfilled grains per panicle (22.8 and 27.0) was observed in weedy check $\left(\mathrm{T}_{11}\right)$ treatment during both years. 
Table.1 Weed density (no. $\left.\mathrm{m}^{-2}\right)$ and dry weight $\left(\mathrm{g} \mathrm{m}^{-2}\right)$ as influenced by different herbicide treatments in direct seeded rice at $30 \mathrm{DAS}$

\begin{tabular}{|c|c|c|c|c|c|c|c|c|c|c|c|c|c|c|c|c|c|c|}
\hline \multirow{3}{*}{\multicolumn{2}{|c|}{ Treatment }} & \multirow{5}{*}{$\begin{array}{c}\text { Dosage } \\
\left(\text { a.i. } \mathbf{g ~ h a}^{-1}\right) \\
20.0+543.0\end{array}$} & \multicolumn{8}{|c|}{ Weed density $\left(\right.$ no. $\left.^{-2}\right)$} & \multicolumn{8}{|c|}{ Weed dry weight $\left(\mathrm{g} \mathrm{m}^{-2}\right)$} \\
\hline & & & \multicolumn{2}{|c|}{ Grasses } & \multicolumn{2}{|c|}{ Sedges } & \multicolumn{2}{|c|}{ Broad leaves } & \multicolumn{2}{|c|}{ Total } & \multicolumn{2}{|c|}{ Grasses } & \multicolumn{2}{|c|}{ Sedges } & \multicolumn{2}{|c|}{ Broad leaves } & \multicolumn{2}{|c|}{ Total } \\
\hline & & & 2017 & 2018 & 2017 & 2018 & 2017 & 2018 & 2017 & 2018 & 2017 & 2018 & 2017 & 2018 & 2017 & 2018 & 2017 & 2018 \\
\hline \multirow[t]{2}{*}{$\mathbf{T}_{1}$} & \multirow[t]{2}{*}{ BS+ 2,4- DSS } & & 2.6 & 3.2 & 1.9 & 1.7 & 2.8 & 2.6 & 4.0 & 4.2 & 1.8 & 2.4 & 1.1 & 1.1 & 2.0 & 1.7 & 2.5 & 2.8 \\
\hline & & & $(5.9)$ & $(9.1)$ & $(2.7)$ & $(2.0)$ & $(6.7)$ & $(5.9)$ & $(15.3)$ & $(17.0)$ & $(2.1)$ & $(4.6)$ & $(0.3)$ & $(0.2)$ & $(3.0)$ & $(2.0)$ & $(5.5)$ & $(6.7)$ \\
\hline \multirow[t]{2}{*}{$\mathbf{T}_{2}$} & \multirow[t]{2}{*}{ BS+ 2,4- DSS } & \multirow[t]{2}{*}{$25.0+678.75$} & 2.3 & 3.0 & 1.9 & 1.7 & 2.7 & 2.4 & 3.7 & 4.0 & 1.5 & 2.3 & 1.1 & 1.0 & 1.9 & 1.6 & 2.3 & 2.6 \\
\hline & & & $(4.1)$ & (7.7) & (2.6) & $(2.0)$ & $(6.3)$ & $(5.0)$ & $(12.9)$ & $(14.7)$ & $(1.2)$ & $(4.3)$ & $(0.3)$ & $(0.0)$ & $(2.7)$ & $(1.4)$ & $(4.1)$ & $(5.7)$ \\
\hline \multirow[t]{2}{*}{$\mathbf{T}_{3}$} & \multirow[t]{2}{*}{ BS+ 2,4- DSS } & \multirow[t]{2}{*}{$30.0+814.5$} & 2.0 & 2.4 & 1.0 & 1.0 & 2.4 & 2.2 & 3.0 & 3.1 & 1.4 & 1.9 & 1.0 & 1.0 & 1.8 & 1.4 & 2.0 & 2.2 \\
\hline & & & $(2.9)$ & $(4.8)$ & $(0.0)$ & $(0.0)$ & $(4.9)$ & $(3.7)$ & $(7.8)$ & $(8.5)$ & $(0.8)$ & $(2.7)$ & $(0.0)$ & $(0.0)$ & $(2.1)$ & $(0.9)$ & $(3.0)$ & $(3.6)$ \\
\hline \multirow[t]{2}{*}{$T_{4}$} & \multirow[t]{2}{*}{$\mathrm{BS}+2,4-\mathrm{DSS}$ (WA) } & \multirow[t]{2}{*}{$20.0+543.0$} & 2.1 & 2.5 & 1.0 & 1.0 & 2.5 & 2.2 & 3.1 & 3.2 & 1.4 & 1.9 & 1.0 & 1.0 & 1.8 & 1.6 & 2.1 & 2.2 \\
\hline & & & (3.3) & $(5.3)$ & $(0.0)$ & $(0.0)$ & $(5.5)$ & (3.9) & $(8.8)$ & $(9.2)$ & $(0.9)$ & (2.6) & $(0.0)$ & $(0.0)$ & $(2.3)$ & (1.4) & $(3.2)$ & $(4.0)$ \\
\hline \multirow[t]{2}{*}{$\mathbf{T}_{5}$} & \multirow{2}{*}{$\mathrm{BS}+2,4-\mathrm{DSS}$ (WA) } & \multirow[t]{2}{*}{$25.0+678.75$} & 1.7 & 2.2 & 1.0 & 1.0 & 2.2 & 2.0 & 2.6 & 2.8 & 1.2 & 1.5 & 1.0 & 1.0 & 1.2 & 1.2 & 1.4 & 1.6 \\
\hline & & & $(1.8)$ & (3.9) & $(0.0)$ & $(0.0)$ & (3.9) & $(2.9)$ & $(5.7)$ & $(6.9)$ & $(0.3)$ & $(1.2)$ & $(0.0)$ & $(0.0)$ & $(0.5)$ & $(0.5)$ & $(0.9)$ & $(1.7)$ \\
\hline$T_{6}$ & $\mathrm{BS}+2,4-\mathrm{DSS}$ (WA) & $30.0+814.5$ & 1.3 & 2.1 & 1.0 & 1.0 & 2.1 & 1.9 & 2.3 & 2.6 & 1.1 & 1.3 & 1.0 & 1.0 & 1.1 & 1.2 & 1.2 & 1.4 \\
\hline & & & $(0.8)$ & $(3.2)$ & $(0.0)$ & $(0.0)$ & (3.6) & $(2.5)$ & $(4.3)$ & $(5.7)$ & $(0.1)$ & $(0.7)$ & $(0.0)$ & $(0.0)$ & $(0.3)$ & $(0.4)$ & $(0.4)$ & $(1.0)$ \\
\hline $\mathbf{T}_{7}$ & Bispyribac sodium $10 \% \mathrm{SC}$ & 20 & 2.7 & 3.3 & 1.9 & 1.8 & 2.9 & 2.9 & 4.2 & 4.5 & 1.8 & 2.5 & 1.2 & 1.1 & 2.1 & 1.8 & 2.7 & 2.9 \\
\hline & & & $(6.3)$ & $(9.8)$ & $(2.8)$ & $(2.3)$ & (7.6) & $(7.2)$ & (16.7) & (19.3) & $(2.2)$ & $(5.1)$ & $(0.4)$ & $(0.1)$ & (3.6) & $(2.3)$ & $(6.2)$ & $(7.5)$ \\
\hline $\mathbf{T}_{8}$ & 2,4- D Ethyl ester $38 \%$ EC ( $34 \%$ & 850 & 3.1 & 3.8 & 2.3 & 2.0 & 3.1 & 3.1 & 4.8 & 5.1 & 2.2 & 3.1 & 1.3 & 1.0 & 2.5 & 2.1 & 3.3 & 3.6 \\
\hline & & & $(8.3)$ & (13.4) & $(4.5)$ & $(2.9)$ & $(8.8)$ & $(8.8)$ & (21.6) & 25.1 & $(3.9)$ & $(8.6)$ & $(0.7)$ & $(0.0)$ & $(5.3)$ & $(3.6)$ & $(9.9)$ & $(12.1)$ \\
\hline $\mathbf{T}_{9}$ & Penoxsulam $21.7 \%$ SC & 22.5 & 2.9 & 3.3 & 1.9 & 1.8 & 3.1 & 3.0 & 4.4 & 4.7 & 1.8 & 2.5 & 1.2 & 1.1 & 2.3 & 2.0 & 2.8 & 3.1 \\
\hline & & & $(7.1)$ & $(10.2)$ & $(2.8)$ & $(2.4)$ & $(8.3)$ & $(8.1)$ & (18.3) & (20.6) & $(2.3)$ & $(5.4)$ & $(0.5)$ & $(0.1)$ & $(4.1)$ & $(2.9)$ & $(6.9)$ & $(8.3)$ \\
\hline$T_{10}$ & Weed free $(20 \& 40$ DAS) & - & 1.0 & 1.0 & 1.0 & 1.0 & 1.0 & 1.0 & 1.0 & 1.0 & 1.0 & 1.0 & 1.0 & 1.0 & 1.0 & 1.0 & 1.0 & 1.0 \\
\hline & & & $(0.0)$ & $(0.0)$ & $(0.0)$ & $(0.0)$ & $(0.0)$ & $(0.0)$ & $(0.0)$ & $(0.0)$ & $(0.0)$ & $(0.0)$ & $(0.0)$ & $(0.0)$ & $(0.0)$ & $(0.0)$ & $(0.0)$ & $(0.0)$ \\
\hline $\mathbf{T}_{11}$ & Weedy check & - & 4.3 & 5.0 & 2.5 & 2.2 & 4.2 & 4.2 & 6.3 & 6.8 & 3.1 & 4.1 & 1.7 & 2.0 & 4.1 & 3.4 & 5.2 & 5.5 \\
\hline & & & $(17.2)$ & $(24.5)$ & $(5.4)$ & $(4.0)$ & (16.4) & $(17.0)$ & $(39.0)$ & $(45.4)$ & $(8.7)$ & $(15.7)$ & (1.9) & $(2.9)$ & (15.6) & (10.8) & $(26.2)$ & $(29.3)$ \\
\hline SEn & & & 0.5 & 0.3 & 0.4 & 0.1 & 0.4 & 0.2 & 0.4 & 0.3 & 0.1 & 0.1 & 0.1 & 0.0 & 0.1 & 0.1 & 0.1 & 0.1 \\
\hline CD & $=0.05$ ) & & 1.4 & 0.7 & 1.1 & 0.4 & 1.1 & 0.6 & 1.3 & 0.8 & 0.4 & 0.3 & 0.3 & 0.1 & 0.3 & 0.3 & 0.3 & 0.4 \\
\hline
\end{tabular}

*DAS: Days after sowing; Figures in parentheses are original values, data were transformed to values $\sqrt{x}+1$ 
Table. 2 Weed density (no. $\left.\mathrm{m}^{-2}\right)$ and dry weight $\left(\mathrm{g} \mathrm{m}^{-2}\right)$ as influenced by different herbicide treatments in direct seeded rice at $60 \mathrm{DAS}$

\begin{tabular}{|c|c|c|c|c|c|c|c|c|c|c|c|c|c|c|c|c|c|c|}
\hline \multirow{3}{*}{\multicolumn{2}{|c|}{ Treatment }} & \multirow{5}{*}{$\begin{array}{c}\text { Dosage } \\
\left(\text { a.i. } \text { g ha }^{-1}\right) \\
20.0+543.0\end{array}$} & \multicolumn{8}{|c|}{ Weed density $\left(\right.$ no. $\left.\mathrm{m}^{-2}\right)$} & \multicolumn{8}{|c|}{ Weed dry weight $\left(\mathrm{g} \mathrm{m}^{-2}\right)$} \\
\hline & & & \multicolumn{2}{|c|}{ Grasses } & \multicolumn{2}{|c|}{ Sedges } & \multicolumn{2}{|c|}{$\begin{array}{l}\text { Broad } \\
\text { leaves }\end{array}$} & \multicolumn{2}{|c|}{ Total } & \multicolumn{2}{|c|}{ Grasses } & \multicolumn{2}{|c|}{ Sedges } & \multicolumn{2}{|c|}{$\begin{array}{l}\text { Broad } \\
\text { leaves }\end{array}$} & \multicolumn{2}{|c|}{ Total } \\
\hline & & & 2017 & 2018 & 2017 & 2018 & 2017 & 2018 & 2017 & 2018 & 2017 & \begin{tabular}{|l|l|}
2018 \\
\end{tabular} & 2017 & 2018 & 2017 & 2018 & 2017 & 2018 \\
\hline \multirow[t]{2}{*}{$\mathbf{T}_{1}$} & \multirow[t]{2}{*}{ BS+ 2,4- DSS } & & 3.7 & 4.7 & 2.3 & 2.2 & 3.5 & 3.1 & 5.4 & 5.8 & 2.1 & 3.0 & 1.4 & 1.2 & 2.5 & 1.9 & 3.2 & 3.5 \\
\hline & & & (13.1) & (20.7) & (4.3) & (3.7) & (11.0) & (8.6) & (28.4) & $(33.0)$ & (3.4) & $(8.2)$ & $(0.9)$ & $(0.4)$ & (5.3) & $(2.5)$ & $(9.5)$ & (11.1) \\
\hline \multirow[t]{2}{*}{$\mathbf{T}_{2}$} & \multirow[t]{2}{*}{$\mathrm{BS}+2,4-\mathrm{DSS}$} & \multirow[t]{2}{*}{$25.0+678.75$} & 3.4 & 4.4 & 2.3 & 2.1 & 3.3 & 3.0 & 5.1 & 5.5 & 2.1 & 3.0 & 1.4 & 1.2 & 2.4 & 1.8 & 3.2 & 3.4 \\
\hline & & & $(10.7)$ & (18.2) & $(4.2)$ & (3.5) & $(9.9)$ & (7.7) & (24.7) & (29.4) & (3.5) & $(7.7)$ & $(0.9)$ & $(0.3)$ & $(4.9)$ & $(2.2)$ & $(9.2)$ & $(10.3)$ \\
\hline \multirow[t]{2}{*}{$\mathbf{T}_{3}$} & \multirow[t]{2}{*}{$\mathrm{BS}+2,4-\mathrm{DSS}$} & \multirow[t]{2}{*}{$30.0+814.5$} & 3.0 & 3.9 & 1.7 & 1.7 & 3.0 & 2.6 & 4.3 & 4.8 & 2.0 & 2.6 & 1.2 & 1.1 & 2.0 & 1.6 & 2.8 & 2.9 \\
\hline & & & $(7.7)$ & (13.9) & $(1.8)$ & $(2.0)$ & $(8.2)$ & $(5.8)$ & (17.7) & (21.7) & (2.9) & (5.6) & $(0.5)$ & $(0.1)$ & $(3.2)$ & $(1.5)$ & (6.6) & $(7.2)$ \\
\hline \multirow[t]{2}{*}{$\mathbf{T}_{4}$} & \multirow[t]{2}{*}{$\mathrm{BS}+2,4-\mathrm{DSS}(\mathrm{WA})$} & \multirow[t]{2}{*}{$20.0+543.0$} & 3.1 & 3.9 & 1.8 & 2.0 & 3.2 & 2.8 & 4.5 & 5.0 & 2.0 & 2.7 & 1.3 & 1.1 & 2.1 & 1.6 & 2.8 & 3.0 \\
\hline & & & $(8.4)$ & (14.4) & $(2.3)$ & $(3.0)$ & $(9.0)$ & $(6.9)$ & (19.7) & $(24.2)$ & (2.9) & $(6.2)$ & $(0.7)$ & $(0.2)$ & $(3.4)$ & (1.6) & $(7.0)$ & $(8.0)$ \\
\hline \multirow[t]{2}{*}{$\mathbf{T}_{5}$} & \multirow[t]{2}{*}{$\mathrm{BS}+2,4-\mathrm{DSS}$ (WA) } & \multirow[t]{2}{*}{$25.0+678.75$} & 2.6 & 3.5 & 1.3 & 1.7 & 2.9 & 2.0 & 3.9 & 4.2 & 1.7 & 2.0 & 1.2 & 1.0 & 1.7 & 1.5 & 2.3 & 2.4 \\
\hline & & & $(6.0)$ & (11.3) & $(0.7)$ & $(2.0)$ & (7.6) & (3.2) & (14.4) & $(16.5)$ & (1.9) & $(3.2)$ & $(0.4)$ & $(0.1)$ & $(2.1)$ & (1.3) & $(4.3)$ & $(4.5)$ \\
\hline$T_{6}$ & $\mathrm{BS}+2,4-\mathrm{DSS}$ (WA) & $30.0+814.5$ & 2.2 & 3.3 & 1.3 & 1.7 & 2.7 & 1.8 & 3.4 & 3.9 & 1.6 & 1.9 & 1.1 & 1.0 & 1.6 & 1.4 & 2.1 & 2.1 \\
\hline & & & (3.9) & $(10.0)$ & $(0.7)$ & $(2.0)$ & $(6.1)$ & $(2.2)$ & $(10.6)$ & (14.3) & $(1.5)$ & (2.6) & $(0.3)$ & $(0.1)$ & $(1.6)$ & $(0.8)$ & (3.4) & $(3.5)$ \\
\hline $\mathbf{T}_{7}$ & Bispyribac sodium $10 \% \mathrm{SC}$ & 20 & 4.0 & 4.8 & 2.4 & 2.2 & 3.7 & 3.3 & 5.7 & 6.1 & 2.2 & 3.2 & 1.5 & 1.2 & 2.6 & 2.0 & 3.4 & 3.7 \\
\hline & & & $(15.0)$ & $(22.3)$ & $(4.6)$ & $(4.0)$ & (12.4) & $(9.7)$ & $(32.1)$ & $(36.0)$ & (3.7) & $(9.0)$ & $(1.1)$ & $(0.4)$ & $(5.7)$ & $(2.9)$ & (10.6) & $(12.4)$ \\
\hline $\mathbf{T}_{8}$ & 2,4- D Ethyl ester $38 \%$ EC ( $34 \%$ & 850 & 5.0 & 5.4 & 2.8 & 2.3 & 4.2 & 3.5 & 6.9 & 6.6 & 2.6 & 3.7 & 1.7 & 1.2 & 2.9 & 2.3 & 4.0 & 4.4 \\
\hline & & & (23.9) & $(28.0)$ & $(6.8)$ & $(4.1)$ & (16.3) & $(10.9)$ & $(46.9)$ & $(43.1)$ & (5.6) & $(13.0)$ & $(2.0)$ & $(0.5)$ & (7.6) & $(4.4)$ & $(15.2)$ & $(17.9)$ \\
\hline $\mathbf{T}_{9}$ & Penoxsulam $21.7 \%$ SC & 22.5 & 4.3 & 4.9 & 2.5 & 2.3 & 3.9 & 3.4 & 6.1 & 6.2 & 2.2 & 3.3 & 1.5 & 1.2 & 2.7 & 2.1 & 3.5 & 3.8 \\
\hline & & & $(17.4)$ & (23.3) & $(5.3)$ & $(4.1)$ & (14.1) & (10.3) & (36.8) & (37.6) & (3.9) & $(9.7)$ & (1.4) & $(0.4)$ & $(6.1)$ & $(3.2)$ & (11.3) & $(13.4)$ \\
\hline$T_{10}$ & Weed free $(20 \& 40$ DAS $)$ & - & 2.0 & 2.3 & 1.0 & 1.0 & 1.1 & 1.5 & 2.1 & 2.5 & 1.0 & 1.3 & 1.0 & 1.0 & 1.1 & 1.1 & 1.1 & 1.3 \\
\hline & & & $(3.0)$ & $(4.3)$ & $(0.0)$ & $(0.0)$ & $(0.3)$ & (1.2) & $(3.2)$ & $(5.5)$ & $(0.1)$ & $(0.6)$ & $(0.0)$ & $(0.0)$ & $(0.1)$ & $(0.2)$ & $(0.2)$ & $(0.8)$ \\
\hline$T_{11}$ & Weedy check & 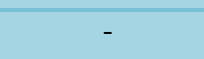 & 5.5 & 6.6 & 3.0 & 2.9 & 5.0 & 4.2 & 7.9 & 8.2 & 4.2 & 5.5 & 2.4 & 2.1 & 5.0 & 3.8 & 6.8 & 6.9 \\
\hline & & & (29.4) & (42.4) & $(8.0)$ & (7.5) & (23.6) & (16.7) & $(60.9)$ & (66.6) & (16.2) & (29.0) & (4.6) & (3.5) & (24.4) & (13.8) & (45.3) & $(46.2)$ \\
\hline SEn & & & 0.7 & 0.3 & 0.5 & 0.2 & 0.5 & 0.2 & 0.5 & 0.3 & 0.2 & 0.2 & 0.0 & 0.0 & 0.2 & 0.1 & 0.2 & 0.1 \\
\hline CD & $=0.05$ ) & & 2.1 & 0.9 & 1.4 & 0.5 & 1.4 & 0.5 & 1.5 & 0.8 & 0.5 & 0.5 & 0.1 & 0.1 & 0.5 & 0.2 & 0.5 & 0.4 \\
\hline
\end{tabular}

*DAS: Days after sowing; Figures in parentheses are original values, data were transformed to values $\sqrt{\mathrm{x}}+1$ 
Table.3 Crop dry matter accumulation, yield attributes, grain and straw yield as influenced by different herbicide treatments in direct seeded rice

\begin{tabular}{|c|c|c|c|c|c|c|c|c|c|c|c|c|c|c|c|c|c|}
\hline \multicolumn{2}{|c|}{ Treatment } & \multirow[t]{2}{*}{$\begin{array}{c}\text { Dosage } \\
\left(\text { a.i. } \mathrm{g} \mathrm{ha}^{-1}\right)\end{array}$} & \multicolumn{3}{|c|}{$\begin{array}{l}\text { Crop dry matter } \\
\text { accumulation } \\
\left(\mathrm{g} \mathrm{m}^{-2}\right)\end{array}$} & \multicolumn{3}{|c|}{$\begin{array}{l}\text { No. of filled grains } \\
\text { panicle }^{-1}\end{array}$} & \multicolumn{3}{|c|}{$\begin{array}{l}\text { No. of unfilled grains } \\
\text { panicle }^{-1}\end{array}$} & \multicolumn{3}{|c|}{$\begin{array}{l}\text { Grain yield (q } \\
\left.\text { ha }^{-1}\right)\end{array}$} & \multicolumn{3}{|c|}{$\begin{array}{l}\text { Straw yield } \\
\left(\mathbf{q} \mathbf{h a}^{-1}\right)\end{array}$} \\
\hline & & & 2017 & 2018 & Mean & 2017 & 2018 & Mean & 2017 & 2018 & Mean & 2017 & 2018 & Mean & 2017 & 2018 & Mean \\
\hline $\begin{array}{l}T \\
1\end{array}$ & $\mathrm{BS}+2,4-\mathrm{DSS}$ & $\begin{array}{c}20.0+ \\
543.0\end{array}$ & 126.3 & 125.2 & 125.7 & 109.0 & 105.5 & 107.3 & 15.5 & 15.5 & 15.5 & 43.9 & 42.9 & 43.4 & 54.4 & 53.8 & 54.1 \\
\hline $\begin{array}{l}T \\
2\end{array}$ & $\mathrm{BS}+2,4-\mathrm{DSS}$ & $\begin{array}{l}25.0+ \\
678.75\end{array}$ & 129.0 & 128.0 & 128.5 & 112.7 & 108.7 & 110.7 & 14.5 & 14.7 & 14.6 & 47.9 & 46.3 & 47.1 & 59.1 & 57.9 & 58.5 \\
\hline $\begin{array}{l}\mathbf{T} \\
3\end{array}$ & $\mathrm{BS}+2,4-\mathrm{DSS}$ & $\begin{array}{l}30.0+ \\
814.5\end{array}$ & 139.1 & 138.0 & 138.6 & 126.7 & 122.8 & 124.8 & 12.0 & 12.7 & 12.3 & 52.0 & 51.1 & 51.6 & 63.8 & 63.4 & 63.6 \\
\hline T & $\mathrm{BS}+2,4-\mathrm{DSS}(\mathrm{WA})$ & $\begin{array}{c}20.0+ \\
543.0\end{array}$ & 139.1 & 137.9 & 138.5 & 125.2 & 121.8 & 123.5 & 12.5 & 12.8 & 12.7 & 51.4 & 49.7 & 50.5 & 63.0 & 62.0 & 62.5 \\
\hline $\begin{array}{l}\mathbf{T} \\
5\end{array}$ & $\mathrm{BS}+2,4-\mathrm{DSS}(\mathrm{WA})$ & $\begin{array}{l}25.0+ \\
678.75\end{array}$ & 140.4 & 139.1 & 139.7 & 129.0 & 124.3 & 126.7 & 11.7 & 12.3 & 12.0 & 54.3 & 52.8 & 53.5 & 66.6 & 65.4 & 66.0 \\
\hline $\begin{array}{l}T \\
6\end{array}$ & $\mathrm{BS}+2,4-\mathrm{DSS}(\mathrm{WA})$ & $\begin{array}{l}30.0+ \\
814.5\end{array}$ & 141.5 & 140.3 & 140.9 & 131.3 & 125.8 & 128.6 & 10.0 & 11.5 & 10.8 & 58.0 & 56.6 & 57.3 & 71.0 & 70.0 & 70.5 \\
\hline $\begin{array}{l}\text { T } \\
7\end{array}$ & Bispyribac sodium $10 \%$ SC & 20 & 125.3 & 124.1 & 124.7 & 106.3 & 104.9 & 105.6 & 17.0 & 15.7 & 16.4 & 40.1 & 38.0 & 39.0 & 49.7 & 49.0 & 49.4 \\
\hline $\begin{array}{l}T \\
8\end{array}$ & $\begin{array}{l}\text { 2,4- D Ethyl ester } 38 \% \text { EC } \\
(34 \% \text { W/W) }\end{array}$ & 850 & 119.6 & 118.5 & 119.0 & 90.7 & 88.7 & 89.7 & 19.7 & 20.0 & 19.8 & 35.7 & 35.3 & 35.5 & 47.3 & 46.2 & 46.8 \\
\hline $\begin{array}{l}\text { T } \\
9\end{array}$ & Penoxsulam $21.7 \%$ SC & 22.5 & 120.7 & 119.4 & 120.0 & 93.0 & 89.7 & 91.3 & 19.2 & 19.8 & 19.5 & 36.3 & 36.1 & 36.2 & 47.6 & 46.5 & 47.0 \\
\hline $\begin{array}{l}T \\
10\end{array}$ & Weed free (20 \& 40 DAS $)$ & - & 143.2 & 142.0 & 142.6 & 132.8 & 126.7 & 129.8 & 9.5 & 11.0 & 10.3 & 59.2 & 57.5 & 58.3 & 72.3 & 70.8 & 71.5 \\
\hline $\begin{array}{c}T \\
11\end{array}$ & Weedy check & - & 107.0 & 105.7 & 106.4 & 79.3 & 73.0 & 76.2 & 22.8 & 27.0 & 24.9 & 29.0 & 28.4 & 28.7 & 38.4 & 38.2 & 38.3 \\
\hline \multicolumn{2}{|c|}{ SEm \pm} & & 1.7 & 1.6 & 1.6 & 2.5 & 1.8 & 2.1 & 1.3 & 0.6 & 1.0 & 2.9 & 2.9 & 2.9 & 3.2 & 3.1 & 3.2 \\
\hline \multicolumn{2}{|c|}{$\mathrm{CD}(\mathrm{P}=0.05)$} & & 4.9 & 4.8 & 4.9 & 7.4 & 5.2 & 6.3 & 3.9 & 1.9 & 2.9 & 8.4 & 8.6 & 8.5 & 9.5 & 9.1 & 9.3 \\
\hline
\end{tabular}


Figure 1(a): Weed control index (\%) of direct seeded rice at 30 DAS as influenced by different herbicides treatment

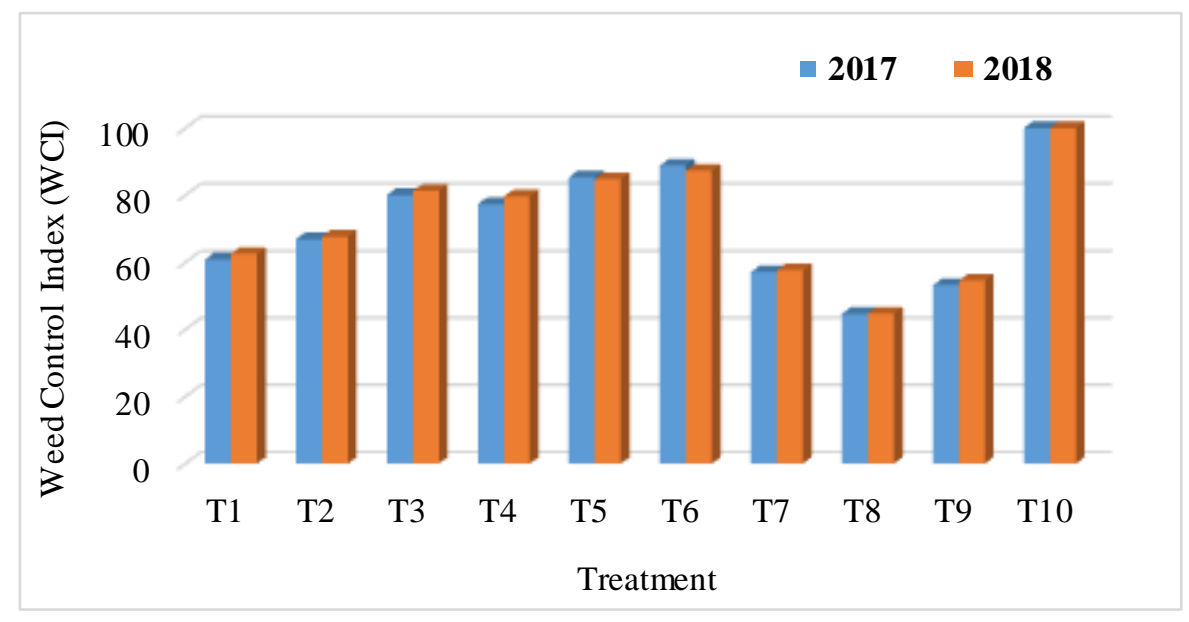

Figure 1(b): Weed control index (\%) of direct seeded rice at 60 DAS as influenced by different herbicides treatment

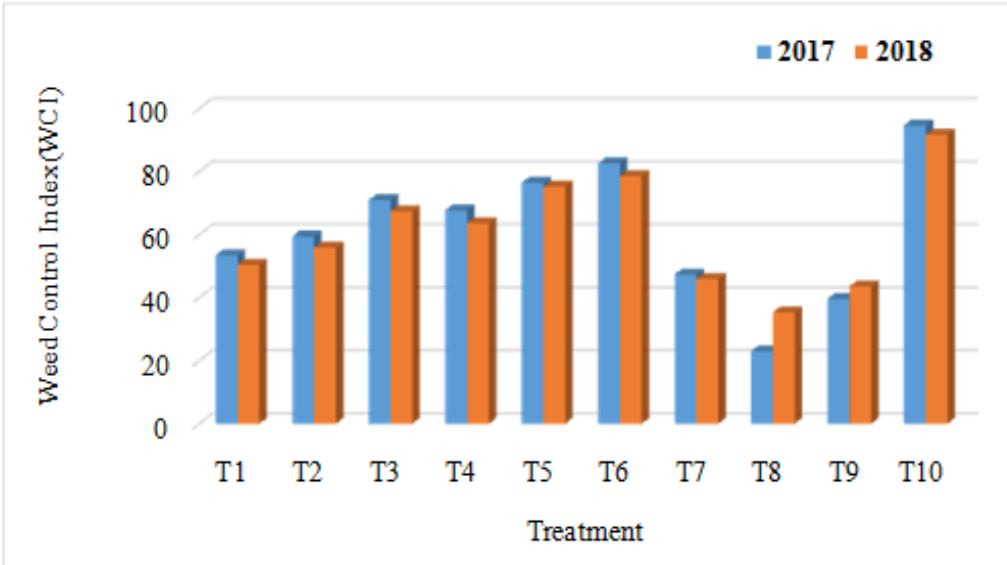

Figure.2 Relationship between no. of filled grains per panicle and no. of unfilled grains per panicle during 2017 (a) and 2018 (b)
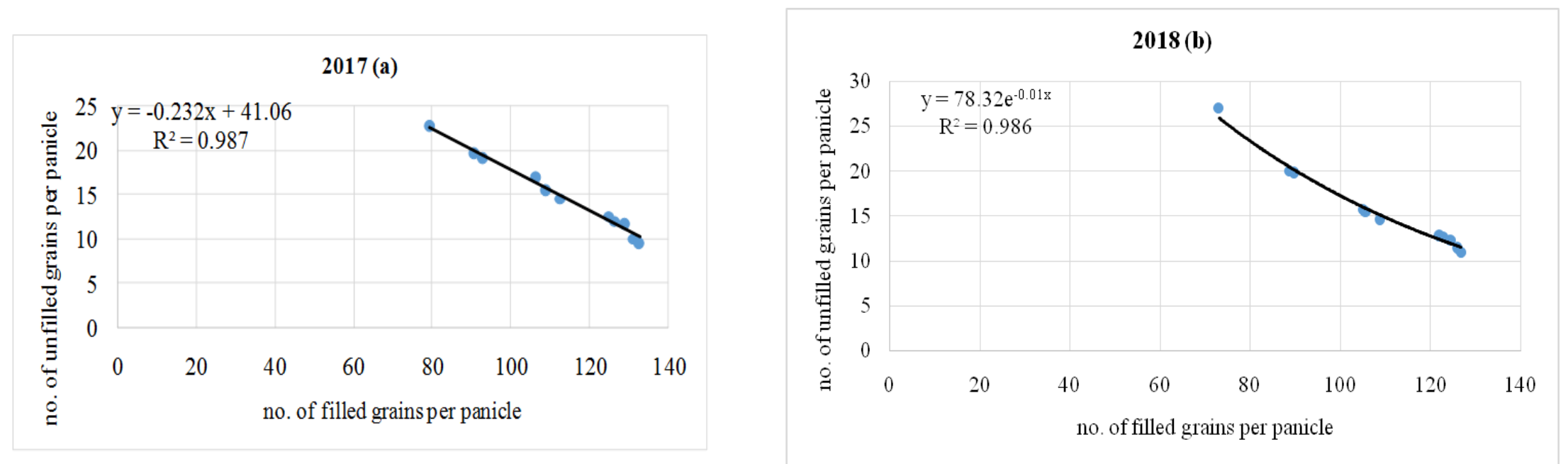
The application of BS+ 2, 4- DSS (WA) $(30.0+$ $\left.814.5 \mathrm{~g} \mathrm{ha}^{-1}\right) \quad\left(\mathrm{T}_{6}\right)$ registered 100.1 and 99.5\%higher grain yield and 56.2 and $57.4 \%$ lower number of unfilled grains per panicle as compare to the weedy check $\left(\mathrm{T}_{11}\right)$ treatment during both years, respectively. These finding were corroborated with Singh et al., (2016). There was strong negative linear relationship between number of filled grains per panicle and number of unfilled grains per panicle in 2017 with $\mathrm{R}^{2}=0.98$ (Fig. 2a) and quadratic relationship in 2018 with $\mathrm{R}^{2}=0.98$ (Fig. 2b). This observation was in consistent to the findings of Choudharyand Dixit (2018).

\section{References}

Anonymous. 2015. Department of Agriculture, Govt. of Chhattisgarh, Raipur. http://agriportal.cg.nic.in/ agridept/AgriHi/KHARIF_15.htm.

Choudhary, V.K. and Dixit, A. 2018. Herbicide weed management on weed dynamics, crop growth and yield in direct-seeded rice. Indian Journal of Weed Science, 50(1): 6-12.

Jacob, G., Menon, M.V. and Abraham, C.T. 2014. Comparative efficacy of new herbicides in direct seeded rice. Journal of Tropical Agriculture, 52(2): 174-177.

Kumar, A., Dhaka, A.K., Kumar, S., Singh, S. and Punia, S.S. 2019. Weed management indices as affected by different weed control treatments in pigeon pea [Cajanus cajan (L.) Millsp.]. Journal of Pharmacognosy and Phytochemistry, 8(3): 3490-3494.

Kumar, V., Bellinder, R.R., Gupta, R.K., Malik, R.K. and Brainard, D.C. 2008. Role of herbicide-resistant rice in promoting resource conservation technologies in rice-wheat cropping systems of India: a review. Crop Protection, 27(5): 290-301.

Mishra, J.S. and Singh, V.P. 2011. Cultivar competitiveness and weed control in zerotill dry-seeded irrigated rice (Oryza sativa L.). The Indian Journal of Agricultural Sciences,81(10): 33-36.

Mooventhan, P., Kadian, K.S., Senthilkumar, R. and Karpagam, C. 2015. Socio-Economic Profiling of Tribal Dairy Farmers in Northern Hills Zone of Chhattisgarh. Journal of Extension Education, 27(3).

Rao, A.N. and Nagamani, A., 2007. Available technologies and future research challenges for managing weeds in dryseeded rice in India. In Proceedings of the 21st Asian Pacific Weed Science Society (APWSS) Conference, 2-6 October 2007, Colombo, Sri Lanka (pp. 391-401). Asian Pacific Weed Science Society.

Singh, S., Bhushan, L., Ladha, J.K., Gupta, R.K., Rao, A.N. and Sivaprasad, B. 2006. Weed management in dry-seeded rice (Oryza sativa) cultivated in the furrowirrigated raised-bed planting system. Crop Protection, 25(5): 487-495.

Singh, V., Jat, M. L., Ganie, Z.A., Chauhan, B. S. and Gupta, R. K. 2016, Herbicide options for effective weed management in dry direct seeded rice under scented ricewheat rotation of western Indo-Gangetic Plains. Crop Protection,81: 168- 176.

Subbaiah, S.V., Singh, S.P., Kumar, R.M. and Padmaja, K. 1999, Drum seeding technology as a substitute for transplanting in India. In 2nd IRRECREMNET Workshop cum Group Meeting. Manila, Philippines Publication: Pp. 24-27.

\section{How to cite this article:}

Satyaraj Guru, R. K. and Sanjay K. Dwivedi. 2020. Assessment of Post-emergence Herbicides in Direct Seeded Rice (Oryza sativa L.) under Chhattisgarh Plain. Int.J.Curr.Microbiol.App.Sci. 9(11): 2699-2706. doi: https://doi.org/10.20546/ijcmas.2020.911.327 